\title{
Analogia da Regra Composicional de Inferência e Operadores Lineares ${ }^{1}$
}

\author{
A.S. CASTILHO ${ }^{2}$, M.E. VALLE ${ }^{3}$, Departamento de Matemática, Centro de Ciências \\ Exatas (CCE), Universidade Estadual de Londrina (UEL), 86051-990, CX.P. 6001, \\ Londrina, PR, Brasil.
}

\begin{abstract}
Resumo. Conjuntos nebulosos são usados para descrever conceitos vagos ou incertos. Sistemas de regras nebulosas (SRNs), por sua vez, é uma poderosa ferramenta matemática para modelar fenômenos usando uma linguagem natural. Métodos de inferência, como o método de Mamdani e a regra composicional de inferência (RCI) de Zadeh, são usados para avaliar um SRNs. Nesse artigo introduzimos os conceitos de espaço reticulado e operadores reticulados, que são análogos aos conceitos de espaço vetorial e operadores lineares. Sobretudo, mostramos que existe uma correspondência unívoca entre operadores reticulados e a RCI. Desse resultado concluímos que RCIs descrevem apenas um subconjunto dos métodos de inferência para SBNs.
\end{abstract}

Palavras-chave. Teoria dos conjuntos nebulosos, sistemas de regras nebulosas, regra composicional de inferência, operadores lineares, reticulados.

\section{Introdução}

Conjuntos nebulosos, também chamados conjuntos fuzzy, representam uma poderosa ferramenta para descrever conceitos vagos e/ou incertos como a noção de pessoa jovem, temperatura confortável e erro pequeno [7, 12, 23]. Além disso, a teoria dos conjuntos nebulosos e a lógica nebulosa oferecem uma metodologia eficiente e transparente para modelagem matemática - transparente no sentido de descrever fenômenos usando uma linguagem natural [18, 25]. De fato, muitos problemas práticos podem ser descritos usando um conjunto de regras nebulosas, i.e., regras da forma "se-então" onde os antecedentes e/ou consequentes são conjuntos nebulosos $[12,24]$. Chamamos método de inferência a técnica usada para fazer conclusões usando um conjunto de regras nebulosas. Aplicações de conjuntos nebulosos inclue dinâmica populacional $[1,9,10]$, diagnóstico [3], controle $[4,13]$, otimização [5] e previsão de séries temporais [21, 22].

É importante esclarecer, entretanto, que a teoria dos conjuntos nebulosos e a lógica nebulosa não são teorias nebulosas ou vagas [18, 25]. Em outras palavras, embora usadas para descrever conceitos que não são claramente definidos, tanto

\footnotetext{
${ }^{1}$ Esse trabalho teve apoio da Coordenação de Aperfeiçoamento de Pessoal de Nível Superior.

${ }^{2}$ E-mail: alexcastilho22@gmail.com.

${ }^{3}$ E-mail: valle@uel.br
} 
a teoria dos conjuntos nebulosos como a lógica nebulosa possuem uma rigorosa base matemática [7, 12]. Especificamente, essas duas teorias podem ser muito bem conduzidas numa estrutura algébrica chamada reticulado [17].

Um reticulado possui duas operações binárias conhecidas por junção e reunião [2]. Nesse artigo, estabelecemos uma analogia entre essas duas operações e as operações usuais de soma e multiplicação. Além disso, apresentamos o conceito de espaço reticulado - um conceito semelhante à noção de espaço vetorial mas definido usando as operações de junção e reunião. Introduzimos também o conceito de operadores reticulados, que são análogos a noção de operador linear e observamos que muitos métodos de inferência, incluindo o método de Mamdani, são exemplos de operadores reticulados $[1,12,18]$. Sobretudo, enunciamos um teorema que caracteriza operadores reticulados. Em termos gerais, tal resultado é análogo ao clássico resultado que afirma que todo operador linear definido sobre espaços finitos corresponde a uma matriz, e vice-versa.

O artigo está organizado da seguinte forma. A próxima seção revisa a teoria dos reticulados e introduz conceitos análogos as noções de espaço vetorial e operador linear. A seção 3, apresenta os conceitos básico da teoria dos conjuntos nebulosos e sistemas de regras nebulosas. Essa seção mostra que muitos métodos de inferência representam operadores reticulados e termina com o teorema que caracteriza esses operadores. O artigo termina com as considerações finais e as referências bibliográficas.

\section{Conceitos Básicos da Teoria dos Reticulados}

A teoria dos reticulados ${ }^{4}$ tem suas origens nos estudos de Dedeking sobre álgebra booleana, no final do século XIX [2]. Muitos outros matemáticos eminentes contribuiram para o desenvolvimento dessa teoria, incluindo Ore, Von Neumann, Tarski e, principalmente, Birkhoff $[2,15,20]$. Essa teoria está baseada apenas em conceitos matemáticos elementares e possui um papel importante em diversas aplicações modernas. Por exemplo, reticulados completos formam a base para muitas técnicas de processamento e análise de imagens $[8,16]$ e inteligência computacional, incluindo redes neurais artificiais e a teoria dos conjuntos nebulosos [7, 11, 17].

Um reticulado é um conjunto parcialmente ordenado onde todo subconjunto finito possue supremo e ínfimo [2]. Denotamos um reticulado por $\mathbb{L}$ ou $\mathbb{M}$. O supremo e o ínfimo de $X \subseteq \mathbb{L}$ serão representados pelos símbolos $\bigvee X$ e $\bigwedge X$, respectivamente. Obtemos um reticulado completo se todo subconjunto, finito ou infinito, possui supremo e ínfimo. O intervalo fechado $[0,1]$ é um exemplo de reticulado completo. O conjunto dos números reais $\mathbb{R}$ representa um reticulado, mas não é um reticulado completo.

As operações de supremo e ínfimo definem duas operações binárias, chamadas junção e reunião ${ }^{5}$, e denotadas respectivamente pelos símbolos $\vee$ e $\wedge$. Especificamente, definimos essas operações através das seguintes equações, para todo $x, y \in \mathbb{L}$ :

$$
x \vee y=\bigvee\{x, y\} \quad \text { e } \quad x \wedge y=\bigwedge\{x, y\} .
$$

\footnotetext{
${ }^{4}$ Tradução do termo inglês "lattice theory".

${ }^{5}$ Tradução dos termos "join" e "meet", respectivamente [2].
} 
Pode-se mostrar que as operações de junção e reunião satisfazem as seguintes propriedades para todo $x, y, z \in \mathbb{L}$ :

1. Comutatividade: $x \vee y=y \vee x$ e $x \wedge y=y \wedge x$.

2. Associatividade: $x \vee(y \vee z)=(x \vee y) \vee z$ e $x \wedge(y \wedge z)=(x \wedge y) \wedge z$.

3. Absorção: $x \wedge(x \vee y)=x \vee(x \wedge y)=x$.

4. Idempotência: $x \vee x=x$ e $x \wedge x=x$.

Reciprocamente, um conjunto equipado com duas operações que satisfazem os 4 itens anteriores é um reticulado [2].

Existe na teoria dos reticulados um importante conceito chamado princípio de dualidade $[2,8]$. Em poucas palavras, esse princípio afirma que existe um reticulado dual associado a todo reticulado $\mathbb{L}$. Com efeito, se $\mathbb{L}$ é um reticulado com uma ordem parcial $\leq$, então $\mathbb{L}$ é também um reticulado com a ordem parcial $\leq^{\prime}$ dada por $x \leq^{\prime} y$ se e somente se $y \leq x$, para todo $x, y \in \mathbb{L}$. O reticularo $\left(\mathbb{L}, \leq^{\prime}\right)$ é chamado dual de $(\mathbb{L}, \leq)$. As operações de junção $\left(\vee^{\prime}\right)$ e reunião $\left(\wedge^{\prime}\right)$ de $\left(\mathbb{L}, \leq^{\prime}\right)$ correspondem às operações de reunião $(\wedge)$ e junção $(\vee)$ do reticulado $(\mathbb{L}, \leq)$. Consequentemente, toda sentença em $(\mathbb{L}, \leq)$ implica uma sentença em $\left(\mathbb{L}, \leq^{\prime}\right)$ que é obtida substituindo as operações de junção por reunião, e vice-versa. As duas equações em (2.2) e (2.3) exemplificam o princípio de dualidade onde a segunda equação corresponde ao dual da primeira. Note que poderíamos definir apenas as primeiras equações em (2.2) e (2.3), e deduzir as demais usando princípio de dualidade. Essa observação será usada nas próximas seções, por exemplo, na definição do espaço reticulado inf- $\vee$.

Dizemos que um reticulado $\mathbb{L}$ é distributivo se as seguintes equações valem para todo $\alpha, x, y \in \mathbb{L}$ :

$$
\alpha \wedge(x \vee y)=(\alpha \wedge x) \vee(\alpha \wedge y) \quad \text { e } \quad \alpha \vee(x \wedge y)=(\alpha \vee y) \wedge(\alpha \vee y)
$$

Analogamente, um reticulado completo $\mathbb{L}$ é infinitamente distributivo se as seguintes equações valem para todo $\alpha \in \mathbb{L}$ e $X \subseteq \mathbb{L}[2,8]$ :

$$
\alpha \wedge(\bigvee X)=\bigvee_{x \in X}(\alpha \wedge x) \quad \text { e } \quad \alpha \vee(\bigwedge X)=\bigwedge_{x \in X}(\alpha \vee x)
$$

\subsection{Espaços reticulados}

As duas Eqs. em 2.2 são análogas a distributividade do produto com a soma, i.e., $\alpha(x+y)=\alpha x+\alpha y$. Por outro lado, as duas Eqs. em 2.3 podem ser identificadas com a equação $\alpha\left(\int_{X} d x\right)=\int_{X}(\alpha d x)$. Desse modo, podemos delinear uma analogia entre as operações de junção e reunião de reticulados com as operações de adição e multiplicação dos números reais. Em vista do princípio de dualidade, podemos identificar sem perda de generalidade, " $\wedge$ " com " $\times "$, " $\vee "$ com "+" e, no caso de reticulados completos, $\int_{X} d x \operatorname{com} \bigvee X$. 
Através das operações de junção " $\vee$ " e reunião " $\wedge$ ", podemos definir a seguinte estrutura algébrica ${ }^{6}$ : Um espaço reticulado sup-^ é um conjunto não vazio $\mathcal{L}$ definido sobre um reticulado distributivo $\mathbb{L}$ onde a operação junção associa a cada par $\mathbf{x}, \mathbf{y} \in \mathcal{L}$ um elemento $\mathbf{x} \vee \mathbf{y} \in \mathcal{L}$ e a operação reunião associa a cada $\mathbf{x} \in \mathcal{L}$ e $\alpha \in \mathbb{L}$ um elemento $\alpha \wedge \mathbf{x} \in \mathcal{L}$. Dualmente, podemos definir o conceito de espaço reticulado inf- $\vee$. Note que os espaços reticulados sup- $\vee$ e inf- $\wedge$ são ambos análogos ao conceito de espaço vetorial, mas definido sobre um reticulado.

Gostaríamos de observar que espaços reticulados também representam reticulados distributivos com operações de junção e reunião $[2,8]$. Em particular, se $\mathbb{L}$ for um reticulado completo infinitamente distributivo então o espaço reticulado $\mathcal{L}$ também representa um reticulado completo infinitamente distributivo.

Exemplo 1. Considere o reticulado completo $\mathbb{L}=[0,1]$. O hipercubo $\mathbb{L}^{n}=[0,1]^{n}$ é um exemplo de espaço reticulado análogo à $\mathbb{R}^{n}$. De fato, dado um escalar $\alpha \in \mathbb{L}$ $e$ vetores coluna $\mathbf{x}=\left[x_{1}, \ldots, x_{n}\right]^{T} \in \mathbb{L}^{n}$ e $\mathbf{y}=\left[y_{1}, \ldots, y_{n}\right]^{T} \in \mathbb{L}^{n}$, podemos definir $\mathbf{x} \vee \mathbf{y}=\left[x_{1} \vee y_{1}, \ldots, x_{n} \vee y_{n}\right]^{T} \in \mathbb{L}^{n}$ e $\alpha \wedge \mathbf{x}=\left[\alpha \wedge x_{1}, \ldots, \alpha \wedge x_{n}\right]^{T} \in \mathbb{L}^{n}$. Neste caso, $\mathbf{x} \vee \mathbf{y}$ é simplesmente o máximo elemento-a-elemento de $\mathbf{x}$ e $\mathbf{y}$. Por outro lado, o vetor $\alpha \wedge \mathbf{x}$ é obtido efetuando um corte de nível $\alpha$ no vetor $\mathbf{x}$. A ordem parcial em $[0,1]^{n}$ é dada por $\mathbf{x} \leq \mathbf{y}$ se, e somente se, $x_{i} \leq y_{i}$ para todo $i=1, \ldots, n$.

Exemplo 2. Dado um conjunto $U$ e o reticulado completo $\mathbb{L}=[0,1]$, o conjunto de todas as funções $\mathbf{x}: U \rightarrow \mathbb{L}$, denotado por $\mathcal{F}(U)$, é um exemplo de espaço reticulado análogo ao espaço das funções continuas definidas sobre um conjunto compacto [14]. Por exemplo, dados $\alpha \in \mathbb{L}$ e funções $\mathbf{x}, \mathbf{y} \in \mathcal{F}(U)$, definimos $\mathbf{x} \vee \mathbf{y} \in \mathcal{F}(U)$ e $\alpha \wedge \mathbf{x} \in \mathcal{F}(U)$ como segue para todo $u \in U:$

$$
(\mathbf{x} \vee \mathbf{y})(u)=\mathbf{x}(u) \vee \mathbf{y}(u) \quad e \quad(\alpha \wedge \mathbf{x})(u)=\alpha \wedge \mathbf{x}(u) .
$$

Finalmente, podemos introduzir um conceito semelhante à noção de operador linear, mas sobre espaços reticulados. Sejam $\mathcal{L}$ e $\mathcal{M}$ espaços reticulados sobre $\mathbb{L}$. Dizemos que $T: \mathcal{L} \rightarrow \mathcal{M}$ é um operador sup- $\wedge$ se a seguinte equação valer para todo $\mathbf{x}, \mathbf{y} \in \mathcal{L}$ e $\alpha, \beta \in \mathbb{L}$ :

$$
T[(\alpha \wedge \mathbf{x}) \vee(\beta \wedge \mathbf{y})]=[\alpha \wedge T(\mathbf{x})] \vee[\beta \wedge T(\mathbf{y})] .
$$

Se $\mathbb{L}$ for um reticulado completo infinitamente distributivo, então um operador sup- $\wedge$ deve satisfazer a seguinte equação para todo $\mathbf{X} \subseteq \mathcal{L}$ e $\alpha \in \mathbb{L}$ :

$$
T(\alpha \wedge(\bigvee \mathbf{X}))=\alpha \wedge\left[\bigvee_{\mathbf{x} \in \mathbf{X}} T(\mathbf{x})\right]
$$

$\mathrm{Na}$ próxima seção mostraremos que os principais sistemas baseados em regras nebulosas representam operadores sup- $\wedge$. Gostaríamos de observar, entretanto, que podemos definir de forma análoga o conceito de operador $i n f-\vee$ aplicando o princípio de dualidade nas Eqs. 2.6 e 2.5. Ambos operadores sup- $\wedge$ e inf- $\vee$ são referidos como operadores reticulados.

\footnotetext{
${ }^{6}$ Nesse artigo, usaremos fontes em negrito para denotar elementos de um espaço reticulado/vetorial e fontes em italico para denotar escalares.
} 


\section{Conceitos Básicos sobre Conjuntos Nebulosos e Sistemas Baseados em Regras Nebulosas}

O conceito de conjunto nebuloso (ou conjunto fuzzy) foi introduzido em 1965 por Zadeh no intuito de descrever matematicamente conceitos vagos empregados frequentemente na linguagem cotidiana [23].

Um conjunto nebuloso, definido sobre um universo de discurso $U$, é caracterizado por uma função de pertinência que atribui um valor no intervalo $[0,1]$ para cada elemento $u \in U$. Em outras palavras, um conjunto nebuloso é descrito por uma função $\mathbf{x}: U \rightarrow[0,1]$. O valor $\mathbf{x}(u)$ representa o grau de compatibilidade de $u \in U$ com o conceito representado por $\mathbf{x}$, i.e., $\mathbf{x}(u)$ representa o quanto $u$ é $\mathbf{x}$. A família de todos os conjuntos nebulosos em $U$ será denotada por $\mathcal{F}(U)$. Lembre-se que $\mathcal{F}(U)$ é um exemplo de espaço reticulado, conforme apresentado no Exemplo 2. Em particular, se $U=\left\{u_{1}, \ldots, u_{n}\right\}$ é um universo de discurso finito, então $\mathbf{x} \in \mathcal{F}(U)$ corresponde à um vetor $\mathbf{x}=\left[x_{1}, \ldots, x_{n}\right]^{T} \in[0,1]^{n}$, onde $x_{i}=\mathbf{x}\left(u_{i}\right)$ para todo $i=1, \ldots, n$. Nesse caso, temos o espaço reticulado apresentado no Exemplo 1.

Na próxima seção mostraremos que alguns métodos de inferência, como o método de Mamdani, representam operadores sup- $\wedge$ [18, 12]. Antes de proseguir, porém, gostaríamos de mencionar que as operações de junção e reunião em $\mathcal{F}(U)$ correspondem às operações de união e intersecção de conjuntos nebulosos [7, 18, 23]. Além disso, as operações " $\wedge$ " e " $\vee$ " correspondem aos conectivos "e" e "ou" na lógica nebulosa, respectivamente $[1,12,17]$.

Finalmente, gostaríamos de lembrar que conjuntos nebulosos definidos sobre o produto cartesiano $U \times V$ de dois universos de discurso são chamados relações nebulosas $[12,17,18]$. Em outras palavras, uma relação nebulosa representa uma função $R: U \times V \rightarrow[0,1]$. A família de todas as relações nebulosas sobre $U \times V$ é denotada por $\mathcal{F}(U \times V)$.

\subsection{Sistemas de regras nebulosas e o método de Mamdani}

Uma regra nebulosa é qualquer sentença da forma se-então onde os antecedentes

e/ou consequentes são conjuntos nebulosos [1, 12, 17, 18]. Várias regras nebulosas formam um Sistema de Regras Nebulosas (SRNs). Matematicamente, podemos representar um SRNs com antecedentes e consequentes nebulosos como segue onde $\mathbf{x}^{\xi} \in \mathcal{F}(U)$ e $\mathbf{y}^{\xi} \in \mathcal{F}(V)$ são conjuntos nebulosos para todo $\xi=1, \ldots, k$.

$$
\text { Se } u \text { é } \mathbf{x}^{\xi} \text { então } v \text { é } \mathbf{y}^{\xi}, \quad \text { para todo } \xi=1, \ldots, k \text {. }
$$

SRNs são usados para modelar problemas que podem ser descritos usando linguagem natural. Aplicações de SRNs inclue avaliação da qualidade de um serviço de transmissão de voz sobre IP, modelagem da evolução de soropositivos para HIV numa determinada população e diagnóstico de cancer de próstata $[1,3,4,9,10]$. Apresentamos abaixo um exemplo simples de SRNs [18].

Exemplo 3. Vamos supor que uma sala possua um aparelho de ar condicionado. Podemos representar a regulagem automatica do aparelho em relação à temperatura 
ambiente através de um SRN com regras do tipo

Se a temperatura é alta, então a potência é máxima,

onde os universos de discurso $U$ e $V$ representam a temperatura ambiente e a potência do aparelho de ar-condicionado, respectivamente. Na regra 3.2, o conjunto nebuloso do antecedente representa o conceito de "temperatura alta" enquanto que o conjunto nebuloso do consequente corresponde à noção de "potência máxima".

É importante observar que regras nebulosas podem ser formuladas de forma simples usando nossa concepção de como o sistema (ar-condicionado) deve funcionar. O SRNs é usado posteriormente para inferir a potência apropriada para uma dada temperatura ambiente.

Dado um SRNs, como em (3.1), e um conjunto nebuloso $\mathrm{x} \in \mathcal{F}(U)$ (e.g. a temperatura ambiente), a forma mais usada para deduzir um conjunto nebuloso $\mathbf{y} \in \mathcal{F}(V)$ (e.g. a potência do ar-condicionado) consiste em efetuar os seguintes passos $[1,12]$ :

1. Calcular o grau de intersecção de $\mathbf{x}$ com os antecedentes $\mathbf{x}^{\xi}$ das regras nebulosas. Em termos matemáticos, calculamos:

$$
r^{\xi}=\bigvee_{u \in U}\left(\mathbf{x}^{\xi}(u) \wedge \mathbf{x}(u)\right), \quad \forall \xi=1, \ldots, k
$$

O valor $r^{\xi}$ mede o grau de compatibilidade dos conjuntos nebulosos $\mathbf{x}$ e $\mathbf{x}^{\xi}$.

2. Definir o conjunto nebuloso $\mathbf{y}$ como a união dos consequentes $\mathbf{y}^{\xi}$ truncados nos respectivos valores $r^{\xi}$, para $\xi=1, \ldots, k$. Especificamente, definimos

$$
\mathbf{y}(v)=\bigvee_{\xi=1}^{k}\left(r^{\xi} \wedge \mathbf{y}^{\xi}(v)\right), \quad \forall v \in V .
$$

A estratégia descrita pelos itens 1 e 2 acima é conhecida como método de inferência de Mamdani [1]. Todavia, é importante lembrar que existem outros métodos de inferência diferente do método proposto por Mamdani [12, 18].

\subsection{Regra composicional de inferência e operadores sup- $\wedge$}

O método de Mamdani representa um caso particular da Regra Composicional de Inferência (RCI), um conceito introduzido nos anos 1970 por Zadeh [24]. Uma RCI define o conjunto nebuloso deduzido y através de uma composição do conjunto nebuloso $\mathrm{x} \in \mathcal{F}(U)$ com uma relação nebulosa $R \in \mathcal{F}(U \times V)$ que captura o comportamento do SRNs. Formalmente, $\mathbf{y} \in \mathcal{F}(V)$ é dado pela equação

$$
\mathbf{y}(v)=T_{R}(\mathbf{x})(v)=\bigvee_{u \in U}(R(u, v) \wedge \mathbf{x}(u)), \quad \forall v \in V .
$$


A relação nebulosa $R \in \mathcal{F}(U \times V)$ pode ser definida de diversas formas [12, 18]. Por exemplo, no método de Mamdani, definimos $R \in \mathcal{F}(U \times V)$ como segue:

$$
R(u, v)=\bigvee_{\xi=1}^{k}\left(\mathbf{x}^{\xi}(u) \wedge \mathbf{y}^{\xi}(v)\right), \quad \text { para todo } u \in U \text { e } v \in V
$$

Note que a Eq. 3.5 define um operador $T_{R}: \mathcal{F}(U) \rightarrow \mathcal{F}(V)$ análogo ao conceito de operador integral, mas definido sobre um espaço reticulado. Lembre-se que um operador integral $T_{K}: \mathcal{C}(U) \rightarrow \mathcal{C}(V)$ é dado pela seguinte equação onde $\mathcal{C}(U)$ e $\mathcal{C}(V)$ denotam o conjunto de todas as funções reais contínuas sobre conjuntos compactos $U$ e $V$, respectivamente, e $K(u, v)$ representa o núcleo do operador integral [14]:

$$
\mathbf{y}(v)=T_{K}(\mathbf{x})(v)=\int_{U} K(u, v) \mathbf{x}(u) d u, \quad \forall v \in V
$$

Operadores integrais são exemplos de operadores lineares sobre o espaço das funções reais contínuas. Portanto, podemos nos perguntar se $T_{R}$ representa um operador reticulado. O seguinte teorema mostra que $T_{R}$ dado pela Eq. 3.5 é um operador sup- $\wedge$. O Teorema 3.1 mostra também que a recíproca é válida, i.e., todo operador sup-^ é descrito pela Eq. 3.5.

Teorema 3.1. Sejam $U$ e $V$ universos de discurso. Um operador $T_{R}: \mathcal{F}(U) \rightarrow$ $\mathcal{F}(V)$ é um operador sup- $\wedge$ se, e somente, se $T_{R}$ é dado pela Eq. 3.5 para alguma relação nebulosa $R \in \mathcal{F}(U \times V)$.

Demonstração. Primeiramente vamos supor que $T_{R}$ seja um operador sup- $\wedge$.

Seja $\mathbf{I}_{u}: U \rightarrow\{0,1\}$ a função indicadora definida como segue: $\mathbf{I}_{u}(\mu)=1$ se $\mu=u$ e $\mathbf{I}_{u}(\mu)=0$ caso contrário. Note que podemos representar $\mathbf{x} \in \mathcal{F}(U)$ como segue usando funções indicadoras:

$$
\mathbf{x}(\mu)=\bigvee_{u \in U}\left(\mathbf{x}(u) \wedge \mathbf{I}_{u}(\mu)\right), \quad \forall \mu \in U
$$

ou, simplesmente,

$$
\mathbf{x}=\bigvee_{u \in U}\left(\mathbf{x}(u) \wedge \mathbf{I}_{u}\right)
$$

Como $T_{R}$ é um operador sup-^, temos

$$
\begin{aligned}
T_{R}(\mathbf{x})(v) & =T_{R}\left(\bigvee_{u \in U}\left(\mathbf{x}(u) \wedge \mathbf{I}_{u}\right)\right)(v)=\bigvee_{u \in U} T_{R}\left(\mathbf{x}(u) \wedge \mathbf{I}_{u}\right)(v) \\
& =\bigvee_{u \in U}\left(\mathbf{x}(u) \wedge\left(T_{R}\left(\mathbf{I}_{u}\right)(v)\right)\right)
\end{aligned}
$$

para todo $v \in V$. Logo $T_{R}$ satisfaz a Eq. 3.5 onde a relação $R \in \mathcal{F}(U \times V)$ é dada por $R(u, v)=T_{R}\left(\mathbf{I}_{u}\right)(v)$ para todo $u \in U$ e $v \in V$. 
Por outro lado, suponha que $T_{R}$ seja dado pela Eq. 3.5, mostraremos que $T_{R}$ é um operador sup- $\wedge$. Para isso, seja $\mathbf{X} \subseteq \mathcal{F}(U)$ e $\alpha \in[0,1]$. Assim, temos

$$
\begin{aligned}
T_{R}(\alpha \wedge(\bigvee \mathbf{X})(v) & =\bigvee_{u \in U}(R(u, v) \wedge(\alpha \wedge(\bigvee \mathbf{X}))(u)) \\
=\alpha \wedge\left[\bigvee_{u \in U}(R(u, v) \wedge(\bigvee \mathbf{X})(u))\right] & =\alpha \wedge\left[\bigvee_{u \in U}\left(\bigvee_{\mathbf{x} \in \mathbf{X}}(R(u, v) \wedge \mathbf{x}(u))\right)\right] \\
=\alpha \wedge\left[\bigvee_{\mathbf{x} \in \mathbf{X}}\left(\bigvee_{u \in U}(R(u, v) \wedge \mathbf{x}(u))\right)\right] & =\alpha \wedge\left[\bigvee_{\mathbf{x} \in \mathbf{X}} T_{R}(\mathbf{x})(v)\right]
\end{aligned}
$$

para todo $v \in V$. Portanto, $T_{R}$ é um operador sup-^ pois satisfaz a Eq. 2.5.

\section{Considerações Finais}

Nesse artigo estabelecemos uma analogia entre as operações de junção e reunião de reticulados com as operações usuais de soma e multiplicação. Além disso, introduzimos conceitos de espaço reticulado, operadores sup- $\wedge$ e inf- $\vee$. Esses conceitos devem ajudar na compreensão e manipulação de conjuntos nebulosos e RCI, pois são análogos às noções de espaço vetorial e operadores lineares. É importante ressaltar, entretanto, que muitos resultados válidos para espaço vetoriais e operadores lineares podem não valer para espaços reticulados e seus operadores. Os conceitos de espaço e operadores reticulados podem apontar novas direções de pesquisa. O leitor interessado em desenvolver pesquisas nesse assunto é convidado à consultar as seguintes referências que tratam de assuntos similares $[2,6,16,19]$.

Finalmente, gostaríamos de observar que muitos livros e artigos sobre a teoria dos conjuntos nebulosos e sobre lógica nebulosa apresentam a Eq. 3.5 como a forma geral de inferência de SNRs com antecedentes e consequentes nebulosos. O argumento usado frequentemente é o seguinte: Como SRNs capturam relações entre as variáveis $u \in U$ e $v \in V$, esses podem então ser representados de forma geral usando uma relação nebulosa. Todavia, o Teorema 3.1 mostra que a Eq. 3.5 descreve apenas um subconjunto de todos os operadores entre as famílias de conjuntos nebulosos $\mathcal{F}(U)$ e $\mathcal{F}(V)$, i.e., a classe dos operadores sup- $\wedge$. O método de inferência introduzido por Kosko constitui um exemplo de operador entre conjuntos nebulosos que não pode ser descrito pela Eq. 3.5 [13, 18]. É importante observar também que a classe dos SRNs funcionais $[12,18]$, que inclue os modelos de Takagi-Sugeno, não faz parte da abordagem apresentada nesse artigo.

\section{Referências}

[1] L. Barros, R. Bassanezi, "Tópicos de Lógica Fuzzy e Biomatemática", Editora do Instituto de Matemática, Estatística e Computação Científica (IMECCUnicamp), Campinas, 2006.

[2] G. Birkhoff, "Lattice Theory", American Mathematical Society, Providence, 3 ed., 1993. 
[3] M. Castanho, L. Barros, A. Yamakami, L. Vendite, Fuzzy expert system: An example in prostate cancer, Applied Mathematics and Computation, 202, No. 1 (2008), 78-85.

[4] A. Castilho, M. Valle, Avaliação da qualidade de um serviço de transimissão de voz sobre ip usando sistemas de regras nebulosas, em "XII Encontro Regional de Matemática Aplicada e Computacional (XII ERMAC)", Foz do Iguaçu, Brasil, 2008. Disponível em: http://www.uel.br/pessoal/valle.

[5] A. Cherri, D. Junior, I. Silva, Uma abordagem fuzzy para o problema de corte de estoque unidimensional com sobras de material aproveitáveis, em "Anais do XXXIX Simpósio Brasileiro de Pesquisa Operacional”, Fortaleza, Brasil, 2007.

[6] R. Cuninghame-Green, "Minimax Algebra: Lecture Notes in Economics and Mathematical Systems", vol. 166, Springer-Verlag, New York, 1979.

[7] D. Dubois, H. Prade, "Fuzzy sets and systems: theory and applications", Academic Press, New York, 1980.

[8] H. Heijmans, "Morphological Image Operators", Academic Press, New York, 1994.

[9] R. Jafelice, L. Barros, R. Bassanezi, F. Gomide, Fuzzy modeling in symptomatic HIV virus infected population, Bulletin of Mathematical Biology, 66, No. 6 (2004), 1597-1620.

[10] R. Jafelice, L. Barros, R. Bassanezi, F. Gomide, Methodology to determine the evolution of asymptomatic HIV population using fuzzy set theory, International Journal of Uncertainty, Fuzziness and Knowledge-Based Systems, 13, No. 1 (2005), 39-58.

[11] V. Kaburlasos, G. Ritter (eds.), "Computational Intelligence Based on Lattice Theory", Springer-Verlag, Heidelberg, Germany, 2007.

[12] G. Klir, B. Yuan, "Fuzzy Sets and Fuzzy Logic: Theory and Applications", Prentice Hall, Upper Saddle River, NY, 1995.

[13] B. Kosko, "Neural Networks and Fuzzy Systems: A Dynamical Systems Approach to Machine Intelligence", Prentice Hall, Englewood Cliffs, NJ, 1992.

[14] E. Kreyszig, "Introductory Functional Analysis with Applications", John Wiley and Sons, 1989.

[15] S. MacLane, Garrett birkhoff and the survey of modern algebra, Notices of the American Mathematical Society, 44, No. 11 (1997), 1438-1439.

[16] P. Maragos, Lattice image processing: A unification of morphological and fuzzy algebraic systems, Journal of Mathematical Imaging and Vision, 22, No. 2-3 (2005), 333-353.

[17] H. Nguyen, E. Walker, "A First Course in Fuzzy Logic', Chapman \& Hall/CRC, Boca Raton, 2 edition, 2000. 
[18] W. Pedrycz, F. Gomide, "Fuzzy Systems Engineering: Toward Human-Centric Computing", Wiley-IEEE Press, New York, 2007.

[19] G. Ritter, P. Gader, Fixed points of lattice transforms and lattice associative memories, in "Advances in Imaging and Electron Physics", (P. Hawkes, ed.) vol. 144. Academic Press, New York, 2006.

[20] G. Rota, The many lives of lattice theory, Notices of the American Mathematical Society, 44, No. 11 (1997), 1440-1445.

[21] P. Sussner, M. Valle, Fuzzy associative memories and their relationship to mathematical morphology, in "Handbook of Granular Computing", (W. Pedrycz, A. Skowron, V. Kreinovich, eds.) pp. 733-754, John Wiley and Sons, Inc., New York, 2008.

[22] M. Valle, "Fundamentos e Aplicações de Memórias Associativas Morfológicas Nebulosas", PhD thesis, Universidade Estadual de Campinas (UNICAMP), Campinas, Brasil, 2007.

[23] L. Zadeh, Fuzzy sets, Information and Control, 8, No. 3 (1965), 338-353.

[24] L. Zadeh, Outline of a new approach to the analysis of complex systems and decision processes, IEEE Transactions on Systems, Man and Cybernetics, SMC-3 No. 1 (1973), 28-44.

[25] L. Zadeh, Is there a need for fuzzy logic? Information Sciences, 178, No. 13 (2008), 2751-2779. 